\title{
PERIODIC SOLUTIONS OF SINGULAR LINEAR AND SEMILINEAR PARABOLIC PROBLEMS*
}

BY

\author{
C. Y. CHAN ${ }^{1}$ AND BENEDICT M. WONG \\ University of Southwestern Louisiana
}

1. Introduction. Let $L_{c}$ be the singular second-order parabolic differential operator defined by

$$
L_{c} u \equiv u_{x x}+\frac{b}{x} u_{x}+c(t) u-u_{t}
$$

where the constant $b<1$ and $c(t) \leq 0$. Recently, Chan and Chen [4] studied quenching phenomena involving $L_{0}$ subject to zero initial data and zero first boundary conditions. The significance of the operator $L_{0}$ is also discussed there. Here, we would like to study periodic solutions for both linear and semilinear problems involving $L_{c}$ subject to first boundary conditions. Periodic solutions for the special case $b=0$ have been studied by many investigators; some more recent results have been obtained by Chan and Wong [5], where further references can be found.

In this work, we are interested in time-periodic solutions of period $T$ (namely, $T$-periodic solutions). We start with investigating the following linear problem:

$$
\begin{array}{cl}
L_{k} u=-\Psi(x, t) & \text { in } \Omega_{\infty}, \\
u(0, t)=0=u(a, t), & -\infty<t<\infty,
\end{array}
$$

where $\Omega_{\infty}=(0, a) \times(-\infty, \infty), k$ is a nonpositive constant, and $\Psi$ is a $T$-periodic function of $t$. In Sec. 2, we make use of a singular Sturm-Liouville problem and the periodic distribution (cf. Zemanian [12, pp. 316-322]) to construct the periodic Green's function $G(x, t ; \xi, \tau)$. In its construction, the presence of the singular term $\frac{b}{x} u_{x}$ gives rise to an infinite series involving products of Bessel functions of the first kind, instead of sine functions for the case $b=0$. This in turn contributes to the difficulties in our present study of both linear and semilinear problems. In Sec. 3, we prove existence of a $T$-periodic solution by establishing its representation formula in terms of the periodic Green's function. We then give existence and uniqueness results for the more general linear problem:

$$
\begin{gathered}
L_{c} u=-\Psi(x, t) \quad \text { in } \Omega_{\infty}, \\
u(0, t)=g_{1}(t), \quad u(a, t)=g_{2}(t), \quad-\infty<t<\infty .
\end{gathered}
$$

${ }^{*}$ Received July $12,1988$.

${ }^{1}$ The work of this author was partially supported by the Board of Regents of the State of Louisiana under Grant LEQSF(86-89)-RD-A-11. 
In Sec. 4 , we begin our study of $T$-periodic solutions of the nonlinear problem:

$$
L_{k} u=-f(x, t, u) \text { in } \Omega_{\infty},
$$

subject to (1.2). We establish its equivalent to an integral equation. In Sec. 5, existence and uniqueness results are given by the monotone method, in contrast to the Picard method used by Liu and Pao [6] for a coupled semilinear parabolic system corresponding to the case $b=0$. The results are then extended to the problem:

$$
L_{c} u=-f(x, t, u) \text { in } \Omega_{\infty},
$$

subject to (1.4).

2. Periodic Green's function. The corresponding homogeneous linear problem of our problem (1.1)-(1.2) is given by

$$
\begin{gathered}
L_{k} v=0 \quad \text { in } \Omega_{\infty}, \\
v(0, t)=0=v(a, t), \quad-\infty<t<\infty .
\end{gathered}
$$

By separation of variables, we obtain from (2.1) and (2.2) the singular Sturm-Liouville problem:

$$
\begin{gathered}
\left(x^{b} X^{\prime}\right)^{\prime}+\lambda x^{b} X=0, \\
X(0)=0=X(a),
\end{gathered}
$$

where $\lambda$ is an eigenvalue. Let $\nu=(1-b) / 2$, which is positive. It follows from McLachlan [7, pp. 116 and 26] that the eigenvalues $\lambda$ are positive, and satisfy $J_{\nu}\left(\lambda^{1 / 2} a\right)=0$, where $J_{\nu}(z)$ is the Bessel function of the first kind of order $\nu$. It is known that $J_{\nu}(z)$ has infinitely many countable zeros for $z>0$; hence, there are infinitely many countable eigenvalues $\lambda_{n}$, which can be arranged in such a way that $\lambda_{1}<\lambda_{2}<\lambda_{3} \cdots$, and $\lambda_{n} \rightarrow \infty$ as $n \rightarrow \infty$ (cf. Watson [10, pp. 490-492]). For each $\lambda_{n}$, let

$$
\phi_{n}(x) \equiv 2^{1 / 2} x^{\nu} J_{\nu}\left(\lambda_{n}^{1 / 2} x\right) /\left(a\left|J_{\nu+1}\left(\lambda_{n}^{1 / 2} a\right)\right|\right) .
$$

Then, $\left\{\phi_{n}(x): n=1,2,3, \ldots\right\}$ forms an orthonormal set of eigenfunctions with weight function $x^{b}$ (cf. McLachlan [7, pp. 102-104]).

Let $\Omega \equiv(0, a) \times(0, T)$, and $\Omega^{-}$be its closure. Let $A$ be a set, $C(A)$ be the set of continuous functions on $A$, and $C^{(m)}(A)$ be the set of $m$-times continuously differentiable functions on $A$. In the sequel, we let $k_{j}(j=1,2,3, \ldots, 26)$ denote appropriate constants.

LEMMA 1.

(i) $\left|\phi_{n}(x)\right| \leq k_{1} x^{-b / 2}$ for $x \in(0, a]$.

(ii) $\left|\phi_{n}(x)\right| \leq k_{2} \lambda_{n}^{1 / 4}$ for $x \in[0, a]$.

(iii) If $x^{b / 2} \psi(x, t)$ is in $C\left(\Omega^{-}\right)$, then

$$
\sum_{n=1}^{\infty}\left[\int_{0}^{a} x^{b} \psi(x, t) \phi_{n}(x) d x\right]^{2} \leq k_{3} \quad \text { for } t \in[0, T] .
$$


(iv) If $x^{b / 2} \psi(x, t)$ in $C\left(\Omega^{-}\right)$is Lipschitz continuous in $t$ uniformly with respect to $x$, then

$$
\sum_{n=1}^{\infty}\left[\frac{d}{d t} \int_{0}^{a} x^{b} \psi(x, t) \phi_{n}(x) d x\right]^{2} \leq k_{4} \quad \text { a.e. on }[0, T] .
$$

Proof. (i) By the asymptotic formula of $J_{\nu}(z)$ (cf. Abramowitz and Stegun [1, p. 364]),

$$
J_{\nu}(z)=\left(\frac{2}{\pi z}\right)^{1 / 2}\left[\cos \left(z-\frac{1}{2} \nu \pi-\frac{1}{4} \pi\right)+O\left(z^{-1}\right)\right]
$$

for $z>0$ and sufficiently large $z$. Because $|\cos x| \leq 1$, and $z^{-1} \rightarrow 0$ as $z \rightarrow \infty$, there exist positive constants $k_{5}$ and $z_{0}$ such that for $z>z_{0}$,

$$
\left|z^{1 / 2} J_{\nu}(z)\right| \leq k_{5} \text {. }
$$

Since $z^{1 / 2} J_{\nu}(z)$ is continuous on $\left[0, z_{0}\right]$, it follows from (2.5) that for $z \geq 0$, there exists a constant $k_{6}$ such that

$$
\left|z^{1 / 2} J_{\nu}(z)\right| \leq k_{6}
$$

By (2.4), we have for large $n$,

$$
\begin{aligned}
& J_{\nu}\left(\lambda_{n}^{1 / 2} a\right)=2^{1 / 2}\left[\cos \left(\lambda_{n}^{1 / 2} a-\frac{1}{2} \nu \pi-\frac{1}{4} \pi\right)+O\left(\lambda_{n}^{-1 / 2} a^{-1}\right)\right] /\left(\pi \lambda_{n}^{1 / 2} a\right)^{1 / 2} \\
& J_{\nu+1}\left(\lambda_{n}^{1 / 2} a\right)=2^{1 / 2}\left[\sin \left(\lambda_{n}^{1 / 2} a-\frac{1}{2} \nu \pi-\frac{1}{4} \pi\right)+O\left(\lambda_{n}^{-1 / 2} a^{-1}\right)\right] /\left(\pi \lambda_{n}^{1 / 2} a\right)^{1 / 2} .
\end{aligned}
$$

Since $J_{\nu}\left(\lambda_{n}^{1 / 2} a\right)=0$, it follows from (2.7) that

$$
\cos \left(\lambda_{n}^{1 / 2} a-\frac{1}{2} \nu \pi-\frac{1}{4} \pi\right) \rightarrow 0 \text { as } n \rightarrow \infty .
$$

Hence,

$$
\left|\sin \left(\lambda_{n}^{1 / 2} a-\frac{1}{2} \nu \pi-\frac{1}{4} \pi\right)\right| \rightarrow 1 \quad \text { as } n \rightarrow \infty .
$$

Therefore, there exists a positive integer $N$ such that for $n>N$,

$$
\left|\sin \left(\lambda_{n}^{1 / 2} a-\frac{1}{2} \nu \pi-\frac{1}{4} \pi\right)+O\left(\lambda_{n}^{-1 / 2} a^{-1}\right)\right| \geq \frac{1}{2} .
$$

From (2.8) and (2.9), we have for $n>N$,

$$
\left|J_{\nu+1}\left(\lambda_{n}^{1 / 2} a\right)\right|^{-1} \leq(2 \pi a)^{1 / 2} \lambda_{n}^{1 / 4} .
$$

Hence, there exists a constant $k_{7}$ such that for any $n$,

$$
\left|J_{\nu+1}\left(\lambda_{n}^{1 / 2} a\right)\right|^{-1} \leq k_{7} \lambda_{n}^{1 / 4} .
$$

By (2.3), (2.6), (2.10), and $b=1-2 \nu$, we have for $x>0$,

$$
\begin{aligned}
\left|\phi_{n}(x)\right| & \leq 2^{1 / 2} k_{7} x^{\nu-1 / 2}\left|\left(\lambda_{n}^{1 / 2} x\right)^{1 / 2} J_{\nu}\left(\lambda_{n}^{1 / 2} x\right)\right| / a \\
& \leq 2^{1 / 2} k_{6} k_{7} x^{\nu-1 / 2} / a \\
& =k_{1} x^{-b / 2}
\end{aligned}
$$

where $k_{1}=2^{1 / 2} k_{6} k_{7} / a$. 
(ii) By Abramowitz and Stegun [1, p. 362], $\left|J_{\nu}\left(\lambda_{n}^{1 / 2} x\right)\right| \leq 1$ for any $n$. Combining this with (2.10), we obtain for any $n$,

$$
\left|\phi_{n}(x)\right| \leq 2^{1 / 2} a^{\nu-1} k_{7} \lambda_{n}^{1 / 4} .
$$

Let $k_{2}=2^{1 / 2} a^{\nu-1} k_{7}$. Then, $\left|\phi_{n}(x)\right| \leq k_{2} \lambda_{n}^{1 / 4}$.

(iii) Since $x^{b / 2} \psi(x, t)$ is in $C\left(\Omega^{-}\right)$and $\Omega^{-}$is compact, it follows that

$$
\int_{0}^{a} x^{b} \psi^{2}(x, t) d x
$$

is bounded by some nonnegative constant $k_{3}$. By Bessel's inequality (cf. Weinberger $[11$, p. 73]),

$$
\sum_{n=1}^{\infty}\left[\int_{0}^{a} x^{b} \psi(x, t) \phi_{n}(x) d x\right]^{2} \leq \int_{0}^{a} x^{b} \psi^{2}(x, t) d x \leq k_{3} .
$$

(iv) By hypothesis, we have for any $x \in[0, a]$,

$$
\left|x^{b / 2}[\psi(x, t+h)-\psi(x, t)]\right| \leq Q_{L}|h|,
$$

where $Q_{L}$ is a Lipschitz constant. Also for each $n, \int_{0}^{a} x^{b} \psi(x, t) \phi_{n}(x) d x$ as a function of $t$ is Lipschitz continuous on [0,T], and hence, it is absolutely continuous and its derivative exists a.e. on $[0, T]$ (cf. Chae [2, pp. 216-217]). By Bessel's inequality and (2.11),

$$
\begin{aligned}
& \sum_{n=1}^{N}\left[\int_{0}^{a} x^{b} h^{-1}[\psi(x, t+h)-\psi(x, t)] \phi_{n}(x) d x\right]^{2} \\
& \quad \leq \int_{0}^{a} x^{b} h^{-2}[\psi(x, t+h)-\psi(x, t)]^{2} d x \\
& \quad \leq a Q_{L}^{2},
\end{aligned}
$$

where $N$ is an integer. As $h \rightarrow 0$, we have

$$
\sum_{n=1}^{N}\left[\frac{d}{d t} \int_{0}^{a} x^{b} \psi(x, t) \phi_{n}(x) d x\right]^{2} \leq a Q_{L}^{2} \quad \text { a.e. on }[0, T] .
$$

Since the above inequality is independent of $N$, Lemma 1 (iv) then follows with $k_{4}=a Q_{L}^{2}$.

We remark that Lemma 1 (iii) and (iv) hold also when the assumptions on $x^{b / 2} \psi(x, t)$ are replaced by the same assumption on $x^{(b+\varepsilon) / 2} \psi(x, t)$, where the constant $\varepsilon$ is less than 1 , since, by similar arguments as in the proofs of Lemma 1 (iii) and (iv), there exist nonnegative constants $k_{8}$ and $k_{9}$ such that

$$
\begin{aligned}
\sum_{n=1}^{\infty}\left[\int_{0}^{a} x^{b} \psi(x, t) \phi_{n}(x) d x\right]^{2} & \leq \int_{0}^{a} x^{-\varepsilon}\left[x^{(b+\varepsilon) / 2} \psi(x, t)\right]^{2} d x \\
& \leq k_{8} \int_{0}^{a} x^{-\varepsilon} d x \\
& =k_{8} a^{1-\varepsilon} /(1-\varepsilon),
\end{aligned}
$$




$$
\begin{aligned}
\sum_{n=1}^{\infty}\left[\frac{d}{d t} \int_{0}^{a} x^{b} \psi(x, t) \phi_{n}(x) d x\right]^{2} & \leq k_{9} \int_{0}^{a} x^{-\varepsilon} d x \\
& =k_{9} a^{1-\varepsilon} /(1-\varepsilon) \text { a.e. on }[0, T]
\end{aligned}
$$

LEMMA 2. If $\left|a_{n}\right| \leq k_{10} \lambda_{n}^{-(3 / 4)-\delta}$, where $\delta$ is a positive constant, then the series $\sum_{n=1}^{\infty} a_{n} \phi_{n}(x)$ converges uniformly on $[0, a]$. The series can be differentiated term by term for $x$ in $(0, a]$ if for positive integer $n,\left|a_{n}\right| \leq k_{11} \lambda_{n}^{-(1+\delta)}$.

Proof. Since $a \lambda_{n}^{1 / 2}$ is the $n$th zero of $J_{\nu}(x)$, it follows from Watson [10, p. 506] that

$$
O\left(\lambda_{n}\right)=O\left(n^{2}\right) \text { for large } n \text {. }
$$

From Lemma 1 (ii), and

$$
\left|a_{n}\right| \leq k_{10} \lambda_{n}^{-(3 / 4)-\delta}
$$

for each $n$, we have

$$
\left|a_{n} \phi_{n}(x)\right| \leq k_{2} k_{10} \lambda_{n}^{-(1 / 2)-\delta} .
$$

From (2.12),

$$
O\left(\left|a_{n} \phi_{n}(x)\right|\right) \leq O\left(n^{-(1+2 \delta)}\right) .
$$

By Weierstrass $M$-test, $\sum_{n=1}^{\infty} a_{n} \phi_{n}(x)$ converges uniformly on [0,a].

By McLachlan [7, p. 192],

$$
\phi_{n}^{\prime}(x)=\left(2 \lambda_{n}\right)^{1 / 2} x^{\nu} J_{\nu-1}\left(\lambda_{n}^{1 / 2} x\right) /\left(a\left|J_{\nu+1}\left(\lambda_{n}^{1 / 2} a\right)\right|\right) .
$$

From (2.4),

$$
J_{\nu-1}\left(\lambda_{n}^{1 / 2} x\right)=\left(\frac{2}{\pi x}\right)^{1 / 2} \lambda_{n}^{-1 / 4}\left\{\cos \left[\lambda_{n}^{1 / 2} x-\frac{1}{2}(\nu-1) \pi-\frac{1}{4} \pi\right]+O\left(\lambda_{n}^{-1 / 2} x^{-1}\right)\right\} .
$$

For $x_{0}>0$, and any $x \in\left[x_{0}, a\right], \lambda_{n}^{-1 / 2} x^{-1} \rightarrow 0$ as $n \rightarrow \infty$. Thus for large $n$, say $n \geq N_{1}$,

$$
\left|\cos \left[\lambda_{n}^{1 / 2} x-\frac{1}{2}(\nu-1) \pi-\frac{1}{4} \pi\right]+O\left(\lambda_{n}^{-1 / 2} x^{-1}\right)\right| \leq 2 .
$$

Hence for $n \geq N_{1}$, and $x \in\left[x_{0}, a\right]$,

$$
\left|J_{\nu-1}\left(\lambda_{n}^{1 / 2} x\right)\right| \leq 2^{3 / 2}(\pi x)^{-1 / 2} \lambda_{n}^{-1 / 4} .
$$

From (2.13), (2.10), and (2.14), it follows that for $n \geq N_{1}$ and $x \in\left[x_{0}, a\right]$,

$$
\left|\phi_{n}^{\prime}(x)\right| \leq 4 k_{7} a^{-1} \pi^{-1 / 2} x^{\nu-1 / 2} \lambda_{n}^{1 / 2} .
$$

Let

$$
\begin{gathered}
k_{12}=\max \left\{\phi_{n}^{\prime}(x) / \lambda_{n}^{1 / 2}: x \in\left[x_{0}, a\right], \text { and } 1 \leq n<N_{1}\right\}, \\
k_{13}=\max \left\{4 k_{7} a^{-1} x_{0}^{\nu-1 / 2}, 4 k_{7} a^{\nu-3 / 2}, k_{12}\right\} .
\end{gathered}
$$

For $x \in\left[x_{0}, a\right]$, and for positive integer $n$,

$$
\left|\phi_{n}^{\prime}(x)\right| \leq k_{13} \lambda_{n}^{1 / 2} .
$$

If $\left|a_{n}\right| \leq k_{11} \lambda_{n}^{-(1+\delta)}$, then

$$
\left|a_{n} \phi_{n}^{\prime}(x)\right| \leq k_{11} k_{13} \lambda_{n}^{-(1 / 2)-\delta}
$$


for $x \in\left[x_{0}, a\right]$. From (2.12),

$$
O\left(\lambda_{n}^{-(1 / 2)-\delta}\right)=O\left(n^{-(1+2 \delta)}\right) .
$$

By Weierstrass $M$-test, $\sum_{n=1}^{\infty} a_{n} \phi_{n}^{\prime}(x)$ converges uniformly on $\left[x_{0}, a\right]$. Hence, $\sum_{n=1}^{\infty} a_{n} \phi_{n}(x)$ can be differentiated term by term for $x \in\left[x_{0}, a\right]$. Since $x_{0}$ is arbitrarily chosen, it follows that $\sum_{n=1}^{\infty} a_{n} \phi_{n}(x)$ can be differentiated term by term for $x \in(0, a]$.

By applying the Theorem in Watson [10, p. 593] to the interval $(0, a)$, we have the following result.

LEMMA 3. If $\int_{0}^{a} x^{b / 2} \varphi(x) d x$ exists (and is absolutely convergent in case the integral is improper), and if $\varphi(x)$ is continuous and of bounded variation on $\left[x_{1}, x_{2}\right]$, where $0<x_{1}<x_{2}<a$, then

$$
\sum_{n=1}^{\infty}\left[\int_{0}^{a} \xi^{b} \varphi(\xi) \phi_{n}(\xi) d \xi\right] \phi_{n}(x)
$$

converges uniformly to $\varphi(x)$ on $\left(x_{1}+\Delta, x_{2}-\Delta\right)$, where $\Delta$ is any positive number.

To construct the periodic Green's function, let us consider the system: for $x$ and $\xi$ in $(0, a)$, and $t$ and $\tau$ in $(-\infty, \infty)$,

$$
\begin{aligned}
L_{k} G(x, t ; \xi, \tau) & =-\delta(x-\xi) \delta_{T}(t-\tau), \\
G(0, t ; \xi, \tau) & =0=G(a, t ; \xi, \tau), \\
G(x, t ; \xi, \tau) & =G(x, t+T ; \xi, \tau),
\end{aligned}
$$

where $\delta(x-\xi)$ is the delta distribution, and $\delta_{T}(t-\tau)$ is the periodic distribution of period $T$ (cf. Zemanian [12, p. 319]). By eigenfunction expansion, let

$$
G(x, t ; \xi, \tau)=\sum_{n=1}^{\infty} a_{n}(t) \phi_{n}(x) .
$$

Since

$$
\phi_{n}^{\prime \prime}(x)+\frac{b}{x} \phi_{n}^{\prime}(x)+\lambda_{n} \phi_{n}(x)=0,
$$

it follows formally that

$$
\sum_{n=1}^{\infty}\left[-a_{n}^{\prime}(t)-\left(\lambda_{n}-k\right) a_{n}(t)\right] \phi_{n}(x)=-\delta(x-\xi) \delta_{T}(t-\tau) .
$$

Multiplying both sides by $x^{b} \phi_{n}(x)$, and integrating from 0 to $a$ with respect to $x$, we have

$$
a_{n}^{\prime}(t)+\left(\lambda_{n}-k\right) a_{n}(t)=\xi^{b} \phi_{n}(\xi) \delta_{T}(t-\tau) .
$$

By Zemanian [12, p. 333],

$$
\delta_{T}(t-\tau)=\frac{1}{T} \sum_{p=-\infty}^{\infty} \exp [i \chi p(t-\tau)]
$$

where $i=(-1)^{1 / 2}$ and $\chi=2 \pi / T$. Integrating

$$
\frac{d}{d t}\left\{a_{n}(t) \exp \left[\left(\lambda_{n}-k\right) t\right]\right\}=\frac{\xi^{b}}{T} \phi_{n}(\xi) \sum_{p=-\infty}^{\infty} \exp \left[\left(\lambda_{n}-k\right) t+i \chi p(t-\tau)\right]
$$


from 0 to $t$, we have formally

$$
\begin{aligned}
a_{n}(t) & \exp \left[\left(\lambda_{n}-k\right) t\right]-a_{n}(0) \\
= & \frac{\xi^{b}}{T} \phi_{n}(\xi) \sum_{p=-\infty}^{\infty} \frac{\exp \left[\left(\lambda_{n}-k\right) t+i \chi p(t-\tau)\right]-\exp (-i \chi p \tau)}{\lambda_{n}-k+i \chi p} .
\end{aligned}
$$

Integrating (2.16) from $t$ to $T$, we obtain formally

$$
\begin{aligned}
a_{n}(T) & \exp \left[\left(\lambda_{n}-k\right) T\right]-a_{n}(t) \exp \left[\left(\lambda_{n}-k\right) t\right] \\
= & \frac{\xi^{b}}{T} \phi_{n}(\xi) \sum_{p=-\infty}^{\infty} \frac{\exp \left[\left(\lambda_{n}-k\right) T-i \chi p \tau\right]-\exp \left[\left(\lambda_{n}-k\right) t+i \chi p(t-\tau)\right]}{\lambda_{n}-k+i \chi p} .
\end{aligned}
$$

The periodic property of Green's function gives $a_{n}(0)=a_{n}(T)$ for any $n$. Adding (2.17) and (2.18), we obtain

$$
a_{n}(0)=\frac{\xi^{b}}{T} \phi_{n}(\xi) \sum_{p=-\infty}^{\infty} \frac{\exp (-i \chi p \tau)}{\lambda_{n}-k+i \chi p}
$$

From (2.17),

$$
a_{n}(t)=\frac{\xi^{b}}{T} \phi_{n}(\xi) \sum_{p=-\infty}^{\infty} \frac{\exp [i \chi p(t-\tau)]}{\lambda_{n}-k+i \chi p} .
$$

Let

$$
E_{n}(y)=\frac{1}{T} \sum_{p=-\infty}^{\infty} \frac{\exp (i \chi p y)}{\lambda_{n}-k+i \chi p}
$$

Then,

$$
G(x, t ; \xi, \tau)=\sum_{n=1}^{\infty} E_{n}(t-\tau) \xi^{b} \phi_{n}(\xi) \phi_{n}(x) .
$$

For $y \in(0, T)$, we expand $\exp \left[-\left(\lambda_{n}-k\right) y\right]$ as complex Fourier series with period $T$. Then,

$$
\exp \left[-\left(\lambda_{n}-k\right) y\right]=\sum_{p=-\infty}^{\infty} \frac{1-\exp \left[-\left(\lambda_{n}-k\right) T\right]}{\left(\lambda_{n}-k+i \chi p\right) T} \exp (i \chi p y)
$$

From (2.19), we have for $y \in(0, T)$,

$$
E_{n}(y)=\frac{\exp \left[-\left(\lambda_{n}-k\right) y\right]}{1-\exp \left[-\left(\lambda_{n}-k\right) T\right]}
$$

Hence, $E_{n}(y)$ is the periodic extension of

$$
\frac{\exp \left[-\left(\lambda_{n}-k\right) y\right]}{1-\exp \left[-\left(\lambda_{n}-k\right) T\right]}
$$

Thus if $(m-1) T<t-\tau<m T$, where $m$ is an integer, we have

$$
E_{n}(t-\tau)=\frac{\exp \left\{-\left(\lambda_{n}-k\right)[t-\tau-(m-1) T]\right\}}{1-\exp \left[-\left(\lambda_{n}-k\right) T\right]} .
$$

It follows that for each $n, E_{n}(t-\tau) \xi^{b} \phi_{n}(\xi) \phi_{n}(x)$ is a $T$-periodic function of $t$ and $\tau$ for $x$ and $\xi$ in $[0, a]$ and $t$ and $\tau$ in $(-\infty, \infty)$. 
If either $\xi$ or $x$ is equal to zero, then

$$
E_{n}(t-\tau) \xi^{b} \phi_{n}(\xi) \phi_{n}(x)=0
$$

since $\phi_{n}(0)=0$ and

$$
\begin{aligned}
\lim _{\xi \rightarrow 0} \xi^{b} \phi_{n}(\xi) & =\lim _{\xi \rightarrow 0} \frac{2^{1 / 2} \xi^{1-\nu}}{a\left|J_{\nu+1}\left(\lambda_{n}^{1 / 2} a\right)\right|}\left[\sum_{r=0}^{\infty} \frac{(-1)^{r}\left(\lambda_{n}^{1 / 2} \xi\right)^{\nu+2 r}}{2^{\nu+2 r}(r !) \Gamma(\nu+r+1)}\right] \\
& =0 .
\end{aligned}
$$

For fixed $x$ and $\xi$ in $(0, a]$, and fixed $t$ and $\tau$, if $(m-1) T<t-\tau<m T$, we have from Lemma 1 (i) and (2.21) that

$$
\left|E_{n}(t-\tau) \xi^{b} \phi_{n}(\xi) \phi_{n}(x)\right| \leq k_{7}^{2} \xi^{b / 2} x^{-b / 2} \frac{\exp \left\{-\left(\lambda_{n}-k\right)[t-\tau-(m-1) T]\right\}}{1-\exp \left[-\left(\lambda_{n}-k\right) T\right]} .
$$

Since $t-\tau-(m-1) T$ is positive and fixed, and $O\left(\lambda_{n}\right)=O\left(n^{2}\right)$ for large $n$, there exists a constant $k_{14}$ such that

$$
\exp \left\{-\left(\lambda_{n}-k\right)[t-\tau-(m-1) T]\right\} \leq \exp \left(-k_{14} n^{2}\right)
$$

for large $n$. Hence,

$$
\sum_{n=1}^{\infty} \frac{\exp \left\{-\left(\lambda_{n}-k\right)[t-\tau-(m-1) T]\right\}}{1-\exp \left[-\left(\lambda_{n}-k\right) T\right]}
$$

converges. Therefore for fixed $x$ and $\xi$ in $(0, a]$, and fixed $t$ and $\tau$ with $t-\tau$ lying between $(m-1) T$ and $m T$,

$$
\sum_{n=1}^{\infty} E_{n}(t-\tau) \xi^{b} \phi_{n}(\xi) \phi_{n}(x)
$$

exists.

From (2.20), $G(x, t ; \xi, \tau)$ exists for any $x$ and $\xi$ in $[0, a]$ and $t$ and $\tau$ in $(-\infty, \infty)$, provided that $t-\tau \neq m T$, where $m$ is an integer. Also, it follows from (2.20) that $G(x, t ; \xi, \tau)$ is a $T$-periodic function of $t$ and $\tau$ since $E_{n}(t-\tau)$ is a $T$-periodic function of $t$ and $\tau$.

\section{Existence and uniqueness for linear problems.}

Definition. The function $u(x, t)$ is said to be a $T$-periodic solution of the problem (1.3)-(1.4) ((1.6) and (1.4)) if $u$ is a $T$-periodic function of $t$ such that

(a) $u$ and $u_{t}$ are in $C\left(\Omega_{\infty}^{-}\right)$,

(b) $u_{x}$ and $u_{x x}$ are in $C\left(\Omega_{\infty}\right)$,

(c) $u(x, t)$ satisfies $(1.3)-(1.4)((1.6)$ and (1.4)), where $\Omega_{\infty}^{-}$denotes the closure of $\Omega_{\infty}$.

We have the following positivity result.

Lemma 4. Let $u(x, t) \in C^{(2)}(\Omega) \cap C\left(\Omega^{-}\right)$, and

$$
\begin{gathered}
L_{\Lambda} u \leq 0 \quad \text { in } \Omega_{\infty}, \\
u(0, t) \geq 0, \quad u(a, t) \geq 0, \quad-\infty<t<\infty, \\
u(x, t)=u(x, t+T), \quad 0<x<a .
\end{gathered}
$$

If $\Lambda(t)$ is continuous and nonpositive, then $u \geq 0$. 
Proof. By the strong maximum principle (cf. Protter and Weinberger [8, pp. 170 and 172]), $u(x, t)$ cannot attain its nonpositive minimum in the interior if $u$ is not a constant. Since $u(x, t)=u(x, t+T)$, the minimum must be attained at $x=0$ or $x=a$. Therefore $u \geq 0$. If $u$ is a constant, then we still have $u \geq 0$ since $u$ is continuous, $u(0, t) \geq 0$, and $u(a, t) \geq 0$.

Let us give an existence result.

THEOREM 5. Suppose $x^{b / 2} \Psi(x, t)$ in $C\left(\Omega^{-}\right)$is a $T$-periodic function of $t$, and is Lipschitz continuous in $t$ uniformly with respect to $x$. If $\Psi(x, t)$ is of bounded variation with respect to $x$ on every given closed subinterval of $(0, a)$, then the problem (1.1)-(1.2) has a $T$-periodic solution $u$ :

$$
u(x, t)=\int_{0}^{T} \int_{0}^{a} G(x, t ; \xi, \tau) \Psi(\xi, \tau) d \xi d \tau .
$$

Proof. Since $G(x, t ; \xi, \tau)$ is periodic, it is clear that $u(x, t)$ is a $T$-periodic function of $t$, provided that the integral in (3.1) exists. From (2.20),

$$
u(x, t)=\int_{0}^{T} \int_{0}^{a} \sum_{n=1}^{\infty} E_{n}(t-\tau) \xi^{b} \phi_{n}(\xi) \phi_{n}(x) \Psi(\xi, \tau) d \xi d \tau .
$$

Let us show that the integral in (3.2) exists, and that the order of integration and summation can be interchanged. Without loss of generality, it is sufficient to consider $t$ in $[0, T]$. Let

$$
E_{n 1}(y)=\exp \left[-\left(\lambda_{n}-k\right) y\right] /\left\{1-\exp \left[-\left(\lambda_{n}-k\right) T\right]\right\}, \quad y>0 .
$$

From (2.20) and (2.21),

$$
\begin{gathered}
G(x, t ; \xi, \tau)=\sum_{n=1}^{\infty} E_{n 1}(t-\tau) \xi^{b} \phi_{n}(\xi) \phi_{n}(x) \quad \text { for } 0<t-\tau<T, \\
G(x, t ; \xi, \tau)=\sum_{n=1}^{\infty} E_{n 1}(T+t-\tau) \xi^{b} \phi_{n}(\xi) \phi_{n}(x) \text { for }-T<t-\tau<0 .
\end{gathered}
$$

By Lemma 1 (i) and (ii),

$$
\left|\xi^{b} \phi_{n}(\xi) \phi_{n}(x)\right| \leq k_{1} k_{2} \lambda_{n}^{1 / 4} \xi^{b / 2} .
$$

Since $\xi^{b / 2} \Psi(\xi, \tau)$ is continuous on $\Omega^{-}$, it follows that $\xi^{b / 2} \Psi(\xi, \tau)$ is bounded. From (3.6), there exists a constant $k_{15}$ such that

$$
\left|\xi^{b} \phi_{n}(\xi) \phi_{n}(x) \Psi(\xi, \tau)\right| \leq k_{15} \lambda_{n}^{1 / 4} .
$$

From (3.3),

$$
\begin{aligned}
\int_{0}^{t} E_{n 1}(t-\tau) d \tau & =\frac{1-\exp \left[-\left(\lambda_{n}-k\right) t\right]}{\left(\lambda_{n}-k\right)\left\{1-\exp \left[-\left(\lambda_{n}-k\right) T\right]\right\}} \\
\int_{t}^{T} E_{n 1}(T+t-\tau) d \tau & =\frac{\exp \left[-\left(\lambda_{n}-k\right) t\right]-\exp \left[-\left(\lambda_{n}-k\right) T\right]}{\left(\lambda_{n}-k\right)\left\{1-\exp \left[-\left(\lambda_{n}-k\right) T\right]\right\}} .
\end{aligned}
$$


Adding (3.8) and (3.9), we have

$$
\int_{0}^{t} E_{n 1}(t-\tau) d \tau+\int_{t}^{T} E_{n 1}(T+t-\tau) d \tau=\frac{1}{\lambda_{n}-k} .
$$

For any fixed $(x, t) \in \Omega^{-}$, let $G_{n}(\xi, \tau)$ be the $n$th partial sum of $G(x, t ; \xi, \tau)$. Then for any fixed $(x, t) \in \Omega^{-}$, the sequence $\left\{G_{n}(\xi, \tau) \Psi(\xi, \tau)\right\}$ converges to $G(x, t ; \xi, \tau) \times$ $\Psi(\xi, \tau)$ a.e. on $\Omega^{-}$. Also from (3.7),

$$
\left|G_{n}(\xi, \tau) \Psi(\xi, \tau)\right| \leq \rho(\tau) \text { for any } n,
$$

where

$$
\rho(\tau)=\left\{\begin{array}{l}
k_{15} \sum_{n=1}^{\infty} \lambda_{n}^{1 / 4} E_{n 1}(t-\tau) \text { for } 0<t-\tau<T, \\
k_{15} \sum_{n=1}^{\infty} \lambda_{n}^{1 / 4} E_{n 1}(T+t-\tau) \text { for }-T<t-\tau<0 .
\end{array}\right.
$$

Let $\rho_{n}(\tau)$ be the $n$th partial sum of $\rho(\tau)$. Then, $\left\{\rho_{n}\right\}$ is a sequence of nonnegative measurable functions converging to $\rho$ with $\rho_{n} \leq \rho$ for any $n$. By the Monotone Convergence Theorem (cf. Royden [9, p. 84]), the Fubini Theorem (cf. Royden [9, p. 269]), and (3.10),

$$
\int_{\Omega} \rho=\lim _{n \rightarrow \infty} \int_{0}^{T} \int_{0}^{a} \rho_{n}(\tau) d \xi d \tau=a k_{15} \sum_{n=1}^{\infty} \lambda_{n}^{1 / 4} /\left(\lambda_{n}-k\right) .
$$

From (2.12), $\sum_{n=1}^{\infty} \lambda_{n}^{1 / 4} /\left(\lambda_{n}-k\right)$ converges. Hence $\rho(\tau)$ is integrable. By the Lebesgue Convergence Theorem (cf. Royden [9, p. 88]) and the Fubini Theorem, we have that, for any fixed $(x, t) \in \Omega^{-}$, the integral exists, and

$$
\begin{aligned}
u(x, t)= & \sum_{n=1}^{\infty} \int_{0}^{t} \int_{0}^{a} \xi^{b} \Psi(\xi, \tau) \phi_{n}(\xi) E_{n 1}(t-\tau) d \xi d \tau \phi_{n}(x) \\
& +\sum_{n=1}^{\infty} \int_{t}^{T} \int_{0}^{a} \xi^{b} \Psi(\xi, \tau) \phi_{n}(\xi) E_{n 1}(T+t-\tau) d \xi d \tau \phi_{n}(x) .
\end{aligned}
$$

By Lemma 1 (iii) and (3.10), there exists a constant $k_{16}$ such that

$$
\begin{aligned}
& \left|\int_{0}^{t} \int_{0}^{a} \xi^{b} \Psi(\xi, \tau) \phi_{n}(\xi) E_{n 1}(t-\tau) d \xi d \tau\right| \\
& \quad+\left|\int_{t}^{T} \int_{0}^{a} \xi^{b} \Psi(\xi, \tau) \phi_{n}(\xi) E_{n 1}(T+t-\tau) d \xi d \tau\right| \leq \frac{k_{16}}{\lambda_{n}-k} .
\end{aligned}
$$

From (3.11) and Lemma 2, it follows that the series representing $u(x, t)$ converges uniformly on $\Omega^{-}$, and hence $u(x, t)$ is in $C\left(\Omega^{-}\right)$.

Next, we would like to show the differentiability of the solution $u(x, t)$. Let

$$
\begin{gathered}
S_{1 m}(x, t) \equiv \sum_{n=1}^{m} \int_{0}^{t} \int_{0}^{a} \xi^{b} \Psi(\xi, \tau) \phi_{n}(\xi) E_{n 1}(t-\tau) d \xi d \tau \phi_{n}(x) \\
S_{2 m}(x, t) \equiv \sum_{n=1}^{m} \int_{t}^{T} \int_{0}^{a} \xi^{b} \Psi(\xi, \tau) \phi_{n}(\xi) E_{n 1}(T+t-\tau) d \xi d \tau \phi_{n}(x) .
\end{gathered}
$$


By Leibnitz's rule of differentiation,

$$
\begin{aligned}
\frac{\partial}{\partial t}\left[S_{1 m}(x, t)+S_{2 m}(x, t)\right] \\
=\sum_{n=1}^{m} \int_{0}^{a} \xi^{b} \Psi(\xi, t) \phi_{n}(\xi) d \xi \phi_{n}(x) \\
\quad-\sum_{n=1}^{m}\left(\lambda_{n}-k\right) \int_{0}^{t} \int_{0}^{a} \xi^{b} \Psi(\xi, \tau) \phi_{n}(\xi) E_{n 1}(t-\tau) d \xi d \tau \phi_{n}(x) \\
\quad-\sum_{n=1}^{m}\left(\lambda_{n}-k\right) \int_{t}^{T} \int_{0}^{a} \xi^{b} \Psi(\xi, \tau) \phi_{n}(\xi) E_{n 1}(T+t-\tau) d \xi d \tau \phi_{n}(x) .
\end{aligned}
$$

Since $\xi^{b / 2} \Psi(\xi, \tau)$ is Lipschitz continuous in $\tau$ uniformly with respect to $\xi$, it follows that for each $n, \int_{0}^{a} \xi^{b} \Psi(\xi, \tau) \phi_{n}(\xi) d \xi$ as a function of $\tau$ is Lipschitz continuous on $[0, T]$, and hence it is absolutely continuous on $[0, T]$. Let

$$
\Psi_{n}(t)=\int_{0}^{a} \xi^{b} \Psi(\xi, t) \phi_{n}(\xi) d \xi
$$

Integrating by parts with respect to $\tau$ (cf. Chae [2, pp. 227-228]) and using $\Psi(\xi, 0)=$ $\Psi(\xi, T)$, we have

$$
\begin{aligned}
\frac{\partial}{\partial t}\left[S_{1 m}(x, t)+S_{2 m}(x, t)\right]= & \sum_{n=1}^{m} \int_{0}^{t} \Psi_{n}^{\prime}(\tau) E_{n 1}(t-\tau) d \tau \phi_{n}(x) \\
& +\sum_{n=1}^{m} \int_{t}^{T} \Psi_{n}^{\prime}(\tau) E_{n 1}(T+t-\tau) d \tau \phi_{n}(x) .
\end{aligned}
$$

From Lemma 1 (iv), (3.10) and Lemma 2,

$$
\lim _{m \rightarrow \infty} \frac{\partial}{\partial t}\left[S_{1 m}(x, t)+S_{2 m}(x, t)\right]
$$

converges uniformly for any $(x, t) \in \Omega^{-}$. Hence, $u(x, t)$ is differentiable with respect to $t$, and

$$
\begin{aligned}
u_{t}(x, t)= & \sum_{n=1}^{\infty} \int_{0}^{t} \Psi_{n}^{\prime}(\tau) E_{n 1}(t-\tau) d \tau \phi_{n}(x) \\
& +\sum_{n=1}^{\infty} \int_{t}^{T} \Psi_{n}^{\prime}(\tau) E_{n 1}(T+t-\tau) d \tau \phi_{n}(x) .
\end{aligned}
$$

Since the series converges uniformly on $\Omega^{-}$and each term in the series is continuous on $\Omega^{-}$, it follows that $u_{t}(x, t)$ is continuous on $\Omega^{-}$. Also from (3.13), we note that $u_{t}(x, 0)=u_{t}(x, T)$. Hence, $u_{t}$ is a $T$-periodic function of $t$. 
Let us show the differentiability of $u(x, t)$ with respect to $x$. Integrating by parts with respect to $\tau$, and using $\Psi(\xi, 0)=\Psi(\xi, T)$ in (3.11), we obtain

$$
\begin{aligned}
u(x, t)= & \sum_{n=1}^{\infty} \Psi_{n}(t) \phi_{n}(x) /\left(\lambda_{n}-k\right) \\
& -\sum_{n=1}^{\infty}\left[\int_{0}^{t} \Psi_{n}^{\prime}(\tau) E_{n 1}(t-\tau) d \tau+\int_{t}^{T} \Psi_{n}^{\prime}(\tau) E_{n 1}(T+t-\tau) d \tau\right] \frac{\phi_{n}(x)}{\lambda_{n}-k} .
\end{aligned}
$$

From Lemma 1 (iv), (3.10) and Lemma 2, the second series can be differentiated term by term with respect to $x$ for $x \in(0, a]$. It follows from (2.15) and Schwarz's inequality that for arbitrarily fixed $x_{0} \in(0, a)$, we have for $x \in\left[x_{0}, a\right]$,

$$
\sum_{n=1}^{\infty}\left|\Psi_{n}(t) \phi_{n}^{\prime}(x) /\left(\lambda_{n}-k\right)\right| \leq k_{13}\left[\sum_{n=1}^{\infty}\left|\Psi_{n}(t)\right|^{2}\right]^{1 / 2}\left[\sum_{n=1}^{\infty} \lambda_{n}\left(\lambda_{n}-k\right)^{-2}\right]^{1 / 2} .
$$

From (3.12), Lemma 1 (iii) and (2.12), there exists a constant $k_{17}$ such that

$$
\left[\sum_{n=1}^{\infty}\left|\Psi_{n}(t)\right|^{2}\right]^{1 / 2}\left[\sum_{n=1}^{\infty} \lambda_{n}\left(\lambda_{n}-k\right)^{-2}\right]^{1 / 2} \leq k_{17}\left[\sum_{n=1}^{\infty} \lambda_{n}\left(\lambda_{n}-k\right)^{-2}\right]^{1 / 2}<\infty .
$$

Therefore,

$$
\sum_{n=1}^{\infty} \Psi_{n}(t) \phi_{n}^{\prime}(x) /\left(\lambda_{n}-k\right)
$$

converges uniformly on $\left[x_{0}, a\right]$, and hence

$$
\sum_{n=1}^{\infty} \Psi_{n}(t) \phi_{n}(x) /\left(\lambda_{n}-k\right)
$$

can be differentiated term by term with respect to $x$ for $x \in\left[x_{0}, a\right]$. Since $x_{0}$ is arbitrarily chosen, it follows that the series can be differentiated term by term with respect to $x$ for $x \in(0, a]$. Thus for $(x, t) \in(0, a] \times[0, T], u_{x}$ is continuous, and

$$
\begin{aligned}
u_{x}(x, t)= & \sum_{n=1}^{\infty} \Psi_{n}(t) \phi_{n}^{\prime}(x) /\left(\lambda_{n}-k\right) \\
& -\sum_{n=1}^{\infty}\left[\int_{0}^{t} \Psi_{n}^{\prime}(\tau) E_{n 1}(t-\tau) d \tau+\int_{t}^{T} \Psi_{n}^{\prime}(\tau) E_{n 1}(T+t-\tau) d \tau\right] \frac{\phi_{n}^{\prime}(x)}{\lambda_{n}-k} .
\end{aligned}
$$

We note that $u_{x}(x, 0)=u_{x}(x, T)$, and hence $u_{x}$ is a $T$-periodic function of $t$.

Let the $m$ th partial sum of $u_{x}(x, t)$ be denoted by $S_{x m}(x, t)$. Using

we have

$$
\phi_{n}^{\prime \prime}(x)+\frac{b}{x} \phi_{n}^{\prime}(x)+\lambda_{n} \phi_{n}(x)=0
$$

$$
\begin{aligned}
\frac{\partial S_{x m}}{\partial x}(x, t)= & -\frac{b}{x} S_{x m}(x, t)-\sum_{n=1}^{m} \Psi_{n}(t) \lambda_{n} \phi_{n}(x) /\left(\lambda_{n}-k\right) \\
& +\sum_{n=1}^{m}\left[\int_{0}^{t} \Psi_{n}^{\prime}(\tau) E_{n 1}(t-\tau) d \tau+\int_{t}^{T} \Psi_{n}^{\prime}(\tau) E_{n 1}(T+t-\tau) d \tau\right] \frac{\lambda_{n} \phi_{n}(x)}{\lambda_{n}-k} .
\end{aligned}
$$


Since the sequence $\left\{S_{x m}(x, t)\right\}$ converges uniformly on $\left[x_{0}, a\right] \times[0, T]$, it follows that the sequence $\left\{\frac{b}{x} S_{x m}(x, t)\right\}$ converges uniformly there. From Lemma 1 (iv) and (3.10), there exists a constant $k_{18}$ such that

$$
\left|\int_{0}^{t} \Psi_{n}^{\prime}(\tau) E_{n 1}(t-\tau) d \tau+\int_{t}^{T} \Psi_{n}^{\prime}(\tau) E_{n 1}(T+t-\tau) d \tau\right| \frac{\lambda_{n}}{\lambda_{n}-k} \leq k_{18} \lambda_{n}^{-1} .
$$

By Lemma 2, the third term on the right-hand side of (3.15) converges uniformly on $\Omega^{-}$as $m \rightarrow \infty$. From (3.12) and Lemma 3, we have for any fixed $t, \sum_{n=1}^{\infty} \Psi_{n}(t) \phi_{n}(x)$ converges uniformly to $\Psi(x, t)$ on $\left[x_{3}, x_{4}\right]$, where $0<x_{3}<x_{4}<\dot{a}$. Hence,

$$
\sum_{n=1}^{\infty} \Psi_{n}(t) \phi_{n}(x) \frac{\lambda_{n}}{\lambda_{n}-k}
$$

converges uniformly on $\left[x_{3}, x_{4}\right]$ for any fixed $t$, and is continuous in $\Omega$. Since each term on the right-hand side of (3.15) converges uniformly on every given closed subinterval of $(0, a)$, it follows that $u_{x}(x, t)$ is differentiable with respect to $x$, and

$$
\begin{aligned}
u_{x x}(x, t)= & -\frac{b}{x} u_{x}(x, t)-\sum_{n=1}^{\infty} \Psi_{n}(t) \lambda_{n} \phi_{n}(x) /\left(\lambda_{n}-k\right) \\
& +\sum_{n=1}^{\infty}\left[\int_{0}^{t} \Psi_{n}^{\prime}(\tau) E_{n 1}(t-\tau) d \tau+\int_{t}^{T} \Psi_{n}^{\prime}(\tau) E_{n 1}(T+t-\tau) d \tau\right] \frac{\lambda_{n} \phi_{n}(x)}{\lambda_{n}-k} .
\end{aligned}
$$

We note that $u_{x x}(x, 0)=u_{x x}(x, T)$, and hence $u_{x x}$ is a $T$-periodic function of $t$. Also since each term on the right-hand side of (3.16) is continuous in $\Omega$, it follows that $u_{x x}(x, t) \in C(\Omega)$.

From (3.13), (3.14), and (3.16),

$$
L_{k} u=-\sum_{n=1}^{\infty} \Psi_{n}(t) \phi_{n}(x) .
$$

By (3.12) and Lemma 3, we have $L_{k} u=-\Psi(x, t)$ for $(x, t) \in(0, a) \times[0, T]$. Since $\phi_{n}(0)=0=\phi_{n}(a)$, it follows from (3.14) that $u(x, t)$ satisfies the homogeneous boundary conditions.

Let us consider the more general problem (1.3)-(1.4). We require the following condition:

(A) $c(t)$ in $C([0, T])$ is a $T$-periodic function.

THEOREM 6. Under condition (A), the problem (1.3)-(1.4) has at most one $T$-periodic solution.

Proof. Suppose $u_{1}(x, t)$ and $u_{2}(x, t)$ are two distinct $T$-periodic solutions. Let $v(x, t)=u_{1}(x, t)-u_{2}(x, t)$. Then, $v(x, t)$ is a $T$-periodic function of $t$ such that

$$
\begin{gathered}
L_{c} v=0, \quad 0<x<a, \quad 0 \leq t<\infty, \\
v(0, t)=0=v(a, t), \quad 0 \leq t<\infty .
\end{gathered}
$$

Without loss of generality, let us assume $v>0$ somewhere. By the strong maximum principle, $v$ attains its positive maximum somewhere at $t=0$. By periodicity, $v$ 
attains this maximum at $t=T$, and we have a contradiction by the strong maximum principle.

The proof of the following result is similar to that of Theorem 2 of Chan and Wong [5] for homogeneous boundary conditions, and hence is omitted here.

Corollary 7. Suppose $\Psi(x, t)$ satisfies the conditions of Theorem 5 and condition (A) holds. Then, the problem (1.3)-(1.4) with $g_{1}(t) \equiv 0 \equiv g_{2}(t)$ has a unique $T$ periodic solution.

We note that it follows from the remark after Lemma 1 that Theorem 5 and Corollary 7 remain valid under the weaker condition:

(B) $x^{(b+\varepsilon) / 2} \Psi(x, t)$ (instead of $\left.x^{b / 2} \Psi(x, t)\right)$ in $C\left(\Omega^{-}\right)$with $0 \leq \varepsilon<1$ is a $T$-periodic function of $t$, and is Lipschitz continuous in $t$ uniformly with respect to $x$.

For nonhomogeneous boundary conditions, we require the following condition:

(C) $g_{1}(t), g_{2}(t), g_{1}^{\prime}(t)$, and $g_{2}^{\prime}(t)$ are $T$-periodic functions in $C([0, T])$ such that $g_{1}^{\prime}(t)$ and $g_{2}^{\prime}(t)$ are Lipschitz continuous over $[0, T]$.

Corollary 8. Under condition (C) and the hypotheses of Corollary 7, if $b>-1$, then the problem (1.3)-(1.4) has a unique $T$-periodic solution.

Proof. Let

$$
\begin{gathered}
\alpha(x, t)=a^{-2 \nu}\left[\left(a^{2 \nu}-x^{2 \nu}\right) g_{1}(t)+x^{2 \nu} g_{2}(t)\right], \\
u(x, t)=V(x, t)+\alpha(x, t),
\end{gathered}
$$

where $V(x, t)$ satisfies

$$
\begin{gathered}
L_{c} V=-\left[\Psi(x, t)+L_{c} \alpha\right] \quad \text { in } \Omega_{\infty}, \\
V(0, t)=0=V(a, t), \quad-\infty<t<\infty .
\end{gathered}
$$

Since $1-2 \nu=b$,

$$
L_{c} \alpha=a^{-2 \nu}\left\{\left(a^{2 \nu}-x^{2 \nu}\right)\left[c(t) g_{1}(t)-g_{1}^{\prime}(t)\right]+x^{2 \nu}\left[c(t) g_{2}(t)-g_{2}^{\prime}(t)\right]\right\} .
$$

Because $b>-1$, it follows that the forcing term $L_{c} \alpha$ satisfies the hypotheses of Theorem 5 under condition (B) with $\Psi(x, t)$ replaced by $L_{c} \alpha$. Thus, $V$ exists and is unique. This implies that $u$ exists, is unique, and

$$
u(x, t)=\left[H_{T}(t)\right]^{-1} \int_{0}^{T} \int_{0}^{a} \bar{G}(x, t ; \xi, \tau) H_{T}(\tau)\left[\Psi(\xi, \tau)+L_{c} \alpha(\xi, \tau)\right] d \xi d \tau+\alpha(x, t),
$$

where

$$
H_{T}(t)=\exp \left\{-\left[\int_{m T}^{t} c(s) d s-(t-m T)\left(\int_{0}^{T} c(s) d s\right) / T\right]\right\}
$$

for any integer $m$ such that $m T \leq t \leq(m+1) T$, and $\bar{G}(x, t ; \xi, \tau)$ is the periodic Green's function $G(x, t ; \xi, \tau)$ with $k$ replaced by $\left(\int_{0}^{T} c(s) d s\right) / T$.

4. Integral equivalent. To show that a $T$-periodic solution of the nonlinear problem (1.5) subject to (1.2) is a $T$-periodic solution of the integral equation,

$$
u(x, t)=\int_{0}^{T} \int_{0}^{a} G(x, t ; \xi, \tau) f(\xi, \tau, u) d \xi d \tau
$$


and conversely, we need the following conditions on $f(x, t, z)$ :

(i) $x^{b / 2} f(x, t, z)$ is in $C\left(\Omega_{\infty}^{-} \times(-\infty, \infty)\right)$;

(ii) $x^{b / 2} f(x, t, z)$ is a $T$-periodic function of $t$ such that

(a) for any $\left(x, t_{1}, z_{1}\right)$ and $\left(x, t_{2}, z_{2}\right)$ in $\Omega^{-} \times\left[\omega_{1}, \omega_{2}\right]$ with $\omega_{1}$ and $\omega_{2}$ being any fixed real numbers,

$$
\left|x^{b / 2}\left[f\left(x, t_{1}, z_{1}\right)-f\left(x, t_{2}, z_{2}\right)\right]\right| \leq Q^{t}\left(\omega_{1}, \omega_{2}\right)\left|t_{1}-t_{2}\right|+Q^{z}\left(\omega_{1}, \omega_{2}\right)\left|z_{1}-z_{2}\right|,
$$

where $Q^{t}\left(\omega_{1}, \omega_{2}\right)$ and $Q^{z}\left(\omega_{1}, \omega_{2}\right)$ are Lipschitz constants depending on the interval $\left[\omega_{1}, \omega_{2}\right]$,

(b) for any $x_{1}$ and $x_{2}$ in $\left[\eta_{1}, \eta_{2}\right] \subset(0, a)$, and $(t, z)$ in $[0, T] \times\left[\omega_{1}, \omega_{2}\right]$,

$$
\left|f\left(x_{1}, t, z\right)-f\left(x_{2}, t, z\right)\right| \leq Q^{x}\left(\eta_{1}, \eta_{2} ; \omega_{1}, \omega_{2}\right)\left|x_{1}-x_{2}\right|
$$

with $Q^{x}\left(\eta_{1}, \eta_{2} ; \omega_{1}, \omega_{2}\right)$ being a Lipschitz constant depending on the intervals $\left[\eta_{1}, \eta_{2}\right]$ and $\left[\omega_{1}, \omega_{2}\right]$.

THEOREM 9. Suppose conditions (i)-(ii) hold. Then, every $T$-periodic solution of the problem (1.5) and (1.2) is a $T$-periodic solution of the integral equation (4.1). Conversely, if $u(x, t)$ is a $T$-periodic solution of the integral equation (4.1), and on $\Omega_{\infty}^{-}, u$ is continuous in $x$, and Lipschitz continuous in $t$ uniformly with respect to $x$, then it is a $T$-periodic solution of the problem (1.5) and (1.2).

Proof. Let us assume that $u(x, t)$ is a $T$-periodic solution of the problem (1.5) and (1.2). Let $F(x, t)=f(x, t, u(x, t))$. Since $u$ is $T$-periodic and continuous on $\Omega_{\infty}^{-}$, there exists a positive integer $N$ such that $|u|<N$. From conditions (i) and (ii), $x^{b / 2} F$ is in $C\left(\Omega^{-}\right)$and is $T$-periodic. From condition (ii) (a),

$$
\left|x^{b / 2}[F(x, t+\zeta)-F(x, t)]\right| \leq Q^{t}(-N, N)|\zeta|+Q^{z}(-N, N)|u(x, t+\zeta)-u(x, t)| .
$$

Since $u$ is $T$-periodic and $u_{t}$ is continuous on $\Omega_{\infty}^{-}$, it follows that $u_{t}$ is $T$-periodic, and hence for any $(x, t) \in \Omega_{\infty}^{-},\left|u_{t}(x, t)\right| \leq k_{19}$ for some constant $k_{19}$. By the Mean Value Theorem,

$$
\left|x^{b / 2}[F(x, t+\zeta)-F(x, t)]\right| \leq\left[Q^{t}(-N, N)+Q^{z}(-N, N) k_{19}\right]|\zeta| .
$$

Therefore, $x^{b / 2} F(x, t)$ is Lipschitz continuous in $t$ uniformly with respect to $x$. Let $\left[\eta_{3}, \eta_{4}\right]$ be any given subinterval of $(0, a)$. From conditions (i) and (ii),

$$
\begin{aligned}
& \sum_{j=1}^{n}\left|F\left(x_{j+1}, t\right)-F\left(x_{j}, t\right)\right| \\
& \quad \leq \sum_{j=1}^{n}\left[Q^{x}\left(\eta_{3}, \eta_{4} ;-N, N\right)\left|x_{j+1}-x_{j}\right|+k_{20} Q^{z}(-N, N)\left|u\left(x_{j+1}, t\right)-u\left(x_{j}, t\right)\right|\right],
\end{aligned}
$$

where $k_{20}$ is a constant such that $\left|x^{-b / 2}\right| \leq k_{20}$ for any $x \in\left[\eta_{3}, \eta_{4}\right]$. Since $u$ is $T$ periodic and $u_{x}$ is in $C\left(\Omega_{\infty}\right)$, it follows that $u_{x}$ is $T$-periodic, and hence for any $(x, t) \in\left[\eta_{3}, \eta_{4}\right] \times(-\infty, \infty),\left|u_{x}\right| \leq k_{21}$ for some constant $k_{21}$. By the Mean Value Theorem,

$$
\sum_{j=1}^{n}\left|F\left(x_{j+1}, t\right)-F\left(x_{j}, t\right)\right| \leq\left[Q^{x}\left(\eta_{3}, \eta_{4} ;-N, N\right)+k_{20} k_{21} Q^{z}(-N, N)\right] \sum_{j=1}^{n}\left|x_{j+1}-x_{j}\right| .
$$


Hence, $F(x, t)$ is of bounded variation with respect to $x$ for every given closed subinterval of $(0, a)$. Since $F(x, t)$ satisfies the conditions of Theorem 5 , it follows that $u(x, t)$ is a $T$-periodic solution of the integral equation (4.1).

Conversely, let $u(x, t)$ be a $T$-periodic solution of the integral equation (4.1) such that on $\Omega_{\infty}^{-}, u$ is continuous in $x$, and Lipschitz continuous in $t$ uniformly with respect to $x$. From conditions (i) and (ii), $x^{b / 2} F(x, t)$ is continuous and periodic. Also, $u(x, t)$ is bounded by a constant $N_{1}$ since $u(x, t)$ is continuous and $T$-periodic on $\Omega_{\infty}^{-}$. By condition (ii) (a), and the hypothesis that $u(x, t)$ is Lipschitz continuous in $t$ uniformly with respect to $x$,

$$
\begin{aligned}
\left|x^{b / 2}[F(x, t+\zeta)-F(x, t)]\right| & \leq Q^{t}\left(-N_{1}, N_{1}\right)|\zeta|+Q^{z}\left(-N_{1}, N_{1}\right)|u(x, t+\zeta)-u(x, t)| \\
& \leq\left[Q^{t}\left(-N_{1}, N_{1}\right)+Q^{z}\left(-N_{1}, N_{1}\right) Q_{u}\right]|\zeta|,
\end{aligned}
$$

where $Q_{u}$ is a Lipschitz constant for $u(x, t)$. Hence, $x^{b / 2} F(x, t)$ is Lipschitz continuous in $t$ uniformly with respect to $x$. By a similar argument as in the proof of Theorem 5, $u$ and $u_{t}$ are in $C\left(\Omega_{\infty}^{-}\right)$, and $u_{x}$ is in $C\left(\Omega_{\infty}\right)$. An argument as in the proof of the first part of this Theorem shows that $F(x, t)$ is of bounded variation with respect to $x$ for every given closed subinterval of $(0, a)$. It follows from Theorem 5 that $u(x, t)$ is a $T$-periodic solution of the problem (1.5) and (1.2).

Let

$$
\beta_{1} \equiv \sup \left\{\sum_{n=1}^{\infty}\left|\phi_{n}(x)\right| /\left(\lambda_{n}-k\right): x \in[0, a]\right\} .
$$

By Lemma 1 (ii) and (2.12), $\beta_{1}$ exists.

Lemma 10. Suppose conditions (i) and (ii) (a) hold. If $u$ is a $T$-periodic solution of the integral equation (4.1) and continuous on $\Omega_{\infty}^{-}$, then there exist constants $\omega_{3}$ and $\omega_{4}$ such that $\omega_{3} \leq u(x, t) \leq \omega_{4}$ for any $(x, t)$ in $\Omega_{\infty}^{-}$; furthermore, $u$ is Lipschitz continuous in $t$ uniformly with respect to $x$, provided that the Lipschitz constant $Q^{z}\left(\omega_{3}, \omega_{4}\right)$ (cf. (4.2)) satisfies

$$
Q^{z}\left(\omega_{3}, \omega_{4}\right) a^{1 / 2} \beta_{1}<1 .
$$

Proof. Since $u$ is continuous on $\Omega_{\infty}^{-}$and $T$-periodic, it follows that $u$ is bounded. Therefore, there exist constants $\omega_{3}$ and $\omega_{4}$ such that $\omega_{3} \leq u(x, t) \leq \omega_{4}$ for any $(x, t)$ in $\Omega_{\infty}^{-}$.

From (2.20), we have

$$
G(x, t+\zeta ; \xi, \tau)=G(x, t ; \xi, \tau-\zeta) .
$$

With $\tau_{1}=\tau-\zeta$,

$$
u(x, t+\zeta)=\int_{-\zeta}^{T-\zeta} \int_{0}^{a} G\left(x, t ; \xi, \tau_{1}\right) f\left(\xi, \tau_{1}+\zeta, u\left(\xi, \tau_{1}+\zeta\right)\right) d \xi d \tau_{1}
$$

Since $G\left(x, t ; \xi, \tau_{1}\right), f\left(\xi, \tau_{1}, z\right)$ and $u\left(\xi, \tau_{1}\right)$ are $T$-periodic functions of $\tau_{1}$, it follows that

$$
u(x, t+\zeta)=\int_{0}^{T} \int_{0}^{a} G(x, t ; \xi, \tau) f(\xi, \tau+\zeta, u(\xi, \tau+\zeta)) d \xi d \tau
$$


Thus,

$$
\begin{aligned}
u(x, t & +\zeta)-u(x, t) \\
= & \int_{0}^{T} \int_{0}^{a} G(x, t ; \xi, \tau)[f(\xi, \tau+\zeta, u(\xi, \tau+\zeta))-f(\xi, \tau, u(\xi, \tau+\zeta))] d \xi d \tau \\
& +\int_{0}^{T} \int_{0}^{a} G(x, t ; \xi, \tau)[f(\xi, \tau, u(\xi, \tau+\zeta))-f(\xi, \tau, u(\xi, \tau))] d \xi d \tau .
\end{aligned}
$$

To complete the proof of the Lemma, it suffices to consider $t$ in $[0, T]$ since $u$ is $T$-periodic. From Bessel's inequality, we have

$$
\begin{aligned}
& \left|\int_{0}^{a} \xi^{b}[f(\xi, \tau+\zeta, u(\xi, \tau+\zeta))-f(\xi, \tau, u(\xi, \tau+\zeta))] \phi_{n}(\xi) d \xi\right| \\
& \leq\left[\int_{0}^{a} \xi^{b}\{f(\xi, \tau+\zeta, u(\xi, \tau+\zeta))-f(\xi, \tau, u(\xi, \tau+\zeta))\}^{2} d \xi\right]^{1 / 2} \\
& \leq a^{1 / 2} Q^{t}\left(\omega_{3}, \omega_{4}\right)|\zeta| \\
& \left|\int_{0}^{a} \xi^{b}[f(\xi, \tau, u(\xi, \tau+\zeta))-f(\xi, \tau, u(\xi, \tau))] \phi_{n}(\xi) d \xi\right| \\
& \quad \leq\left[\int_{0}^{a} \xi^{b}\{f(\xi, \tau, u(\xi, \tau+\zeta))-f(\xi, \tau, u(\xi, \tau))\}^{2} d \xi\right]^{1 / 2} \\
& \leq a^{1 / 2} Q^{z}\left(\omega_{3}, \omega_{4}\right)\|u\|_{\zeta},
\end{aligned}
$$

where

$$
\|u\|_{\zeta}=\max \left\{|u(\xi, \tau+\zeta)-u(\xi, \tau)|:(\xi, \tau) \in \Omega^{-}\right\} .
$$

By (4.4), (3.4), (3.5), and (3.10),

$$
\begin{aligned}
\|u\|_{\zeta} \leq & {\left[Q^{t}\left(\omega_{3}, \omega_{4}\right)|\zeta|+Q^{z}\left(\omega_{3}, \omega_{4}\right)\|u\|_{\zeta}\right] a^{1 / 2} } \\
& \times \sum_{n=1}^{\infty}\left|\phi_{n}(x)\right|\left[\int_{0}^{t} E_{n 1}(t-\tau) d \tau+\int_{t}^{T} E_{n 1}(T+t-\tau) d \tau\right] \\
= & {\left[Q^{t}\left(\omega_{3}, \omega_{4}\right)|\zeta|+Q^{z}\left(\omega_{3}, \omega_{4}\right)\|u\|_{\zeta}\right] a^{1 / 2} \sum_{n=1}^{\infty}\left|\phi_{n}(x)\right| /\left(\lambda_{n}-k\right) . }
\end{aligned}
$$

Hence from (4.3),

$$
\left[1-Q^{z}\left(\omega_{3}, \omega_{4}\right) a^{1 / 2} \beta_{1}\right]\|u\|_{\zeta} \leq Q^{t}\left(\omega_{3}, \omega_{4}\right) a^{1 / 2} \beta_{1}|\zeta| .
$$

By hypothesis,

$$
1-Q^{z}\left(\omega_{3}, \omega_{4}\right) a^{1 / 2} \beta_{1}>0
$$

Thus,

$$
\|u\|_{\zeta} \leq\left\{Q^{t}\left(\omega_{3}, \omega_{4}\right) a^{1 / 2} \beta_{1} /\left[1-Q^{z}\left(\omega_{3}, \omega_{4}\right) a^{1 / 2} \beta_{1}\right]\right\}|\zeta|,
$$

and hence $u(x, t)$ is Lipschitz continuous in $t$ uniformly with respect to $x$.

5. Monotone method. We assume that conditions (i)-(ii) hold. To use the monotone method (cf. Chan [3]) to establish existence, we require the following conditions:

(D) $\gamma(x, t)$ in $C^{(2)}\left(\Omega_{\infty}^{-}\right)$satisfies the same conditions for $\Psi$ in Theorem 5 such that $L_{0} \gamma \geq 0$ and $\gamma(x, t) \leq 0$ 
(E) for each $\omega>0$, there exists a bounded and nonnegative function $q_{\omega}(x, t)$ such that for $|u| \leq \omega$,

$$
-f(x, t, u(x, t)) \leq q_{\omega}(x, t)[u(x, t)-\gamma(x, t)]
$$

if $u(x, t) \geq \gamma(x, t)$

(F) there exists a nonnegative function $\sigma(x, t)$ such that $\sigma(x, t)$ satisfies the same conditions for $\Psi$ in Theorem 5 , and

$$
-f(x, t, u(x, t)) \geq-\sigma(x, t)
$$

if $u(x, t) \geq \gamma(x, t)$;

(G) for each $\omega>0$, there exists a bounded and nonnegative function $p_{\omega}(x, t)$ such that for $|u| \leq \omega$ and $|v| \leq \omega$,

$$
-[f(x, t, u(x, t))-f(x, t, v(x, t))] \leq p_{\omega}(x, t)[u(x, t)-v(x, t)]
$$

if $u(x, t) \geq v(x, t)$;

(H) $-f(x, t, u(x, t)) \geq-f(x, t, v(x, t))$ if $u(x, t) \geq v(x, t)$ for any bounded functions $u$ and $v$ greater than or equal to $\gamma$.

We remark that, in particular, $\gamma$ or $\sigma$ can be taken to be identically zero.

Let $M_{0}(x, t)$ be constructed by

$$
\begin{gathered}
L_{k} M_{0}=-\sigma(x, t) \quad \text { in } \Omega_{\infty}, \\
M_{0}(0, t)=0=M_{0}(a, t), \quad-\infty<t<\infty .
\end{gathered}
$$

By Theorems 5 and $6, M_{0}(x, t)$ exists, is unique, and

$$
M_{0}(x, t)=\int_{0}^{T} \int_{0}^{a} G(x, t ; \xi, \tau) \sigma(\xi, \tau) d \xi d \tau .
$$

By conditions (D) and (F),

$$
\begin{gathered}
\quad L_{k}\left(M_{0}-\gamma\right)=-\sigma(x, t)-L_{k} \gamma \leq 0 \quad \text { in } \Omega_{\infty}, \\
\left(M_{0}-\gamma\right)(0, t)=-\gamma(0, t) \geq 0, \quad\left(M_{0}-\gamma\right)(a, t)=-\gamma(a, t) \geq 0, \quad-\infty<t<\infty .
\end{gathered}
$$

By Lemma $4, M_{0}(x, t) \geq \gamma(x, t)$. Since $\gamma(x, t)$ in $C^{(2)}\left(\Omega_{\infty}^{-}\right)$and $M_{0}(x, t)$ in $C\left(\Omega_{\infty}^{-}\right)$ are $T$-periodic, there exist constants $k_{22}$ and $k_{23}$ such that

$$
k_{22} \leq \gamma(x, t) \leq M_{0}(x, t) \leq k_{23}
$$

Let $\omega^{\prime}=\max \left\{\left|k_{22}\right|,\left|k_{23}\right|\right\}$. By conditions $(\mathrm{G})$ and $(\mathrm{E})$, both $p_{\omega^{\prime}}(x, t)$ and $q_{\omega^{\prime}}(x, t)$ are bounded, and hence there exists a nonnegative constant $\mu$ such that

(a) if

$$
k_{22} \leq v(x, t) \leq u(x, t) \leq k_{23},
$$

then

$$
-[f(x, t, u(x, t))-f(x, t, v(x, t))] \leq \mu[u(x, t)-v(x, t)] ;
$$

(b) if $k_{22} \leq u(x, t) \leq k_{23}$, and $u(x, t) \geq \gamma(x, t)$, then

$$
-f(x, t, u(x, t)) \leq \mu[u(x, t)-\gamma(x, t)] .
$$


Theorem 11. Suppose conditions (D)-(G) hold. Then, the sequence $\left\{M_{j+1}(x, t)\right.$ : $j=0,1,2, \ldots\}$ constructed by

$$
\begin{gathered}
L_{k} M_{j+1}=-f\left(x, t, M_{j}(x, t)\right)+\mu\left[M_{j+1}(x, t)-M_{j}(x, t)\right] \text { in } \Omega_{\infty}, \\
M_{j+1}(0, t)=0=M_{j+1}(a, t), \quad-\infty<t<\infty,
\end{gathered}
$$

satisfies the inequalities:

$$
k_{22} \leq \gamma(x, t) \leq M_{j+1}(x, t) \leq M_{j}(x, t) \leq M_{0}(x, t) \leq k_{23} \text { on } \Omega_{\infty}^{-} ;
$$

furthermore, $\left\{M_{j+1}\right\}$ converges uniformly to $M ; M$ is continuous on $\Omega_{\infty}^{-}$, and is a $T$ periodic solution of the integral equation (4.1). Also, $M \geq u$, where $u$ is a $T$-periodic solution of the problem (1.5) and (1.2) with $u \geq \gamma$.

Proof. By construction,

$$
\begin{gathered}
\left(L_{k}-\mu\right) M_{1}=-f\left(x, t, M_{0}\right)-\mu M_{0} \quad \text { in } \Omega_{\infty}, \\
M_{1}(0, t)=0=M_{1}(a, t), \quad-\infty<t<\infty .
\end{gathered}
$$

Since $M_{0}$ and $\partial M_{0} / \partial t$ are in $C\left(\Omega_{\infty}^{-}\right), \partial M_{0} / \partial x$ is in $C\left(\Omega_{\infty}\right)$, and $\partial M_{0} / \partial t$ and $\partial M_{0} / \partial x$ are periodic, it follows from an argument similar to the proof of the first part of Theorem 9 that $-f\left(x, t, M_{0}\right)-\mu M_{0}$ satisfies the hypotheses of Theorem 5 . Thus, $M_{1}(x, t)$ exists and is unique. By condition $(\mathrm{F})$,

$$
\begin{gathered}
\left(L_{k}-\mu\right)\left(M_{0}-M_{1}\right)=-\left[\sigma(x, t)-f\left(x, t, M_{0}(x, t)\right)\right] \leq 0 \quad \text { in } \Omega_{\infty}, \\
\left(M_{0}-M_{1}\right)(0, t)=0=\left(M_{0}-M_{1}\right)(a, t), \quad-\infty<t<\infty .
\end{gathered}
$$

From Lemma 4, $M_{0} \geq M_{1}$. By condition (D) and (5.2),

$$
\begin{gathered}
\left(L_{k}-\mu\right)\left(M_{1}-\gamma\right)=-f\left(x, t, M_{0}\right)-\mu\left(M_{0}-\gamma\right)-L_{k} \gamma \leq 0 \quad \text { in } \Omega_{\infty}, \\
\left(M_{1}-\gamma\right)(0, t)=-\gamma(0, t) \geq 0, \quad\left(M_{1}-\gamma\right)(a, t)=-\gamma(a, t) \geq 0, \quad-\infty<t<\infty .
\end{gathered}
$$

From Lemma $4, M_{1} \geq \gamma$. Thus, we have the inequalities:

$$
k_{22} \leq \gamma \leq M_{1} \leq M_{0} \leq k_{23} .
$$

Similarly, $M_{2}$ exists and is unique. Let us assume

$$
k_{22} \leq \gamma \leq M_{q} \leq M_{q-1} \leq \cdots \leq M_{0} \leq k_{23}
$$

for some positive integer $q$. By (5.1),

$\left(L_{k}-\mu\right)\left(M_{q}-M_{q+1}\right)=-\left[f\left(x, t, M_{q-1}\right)-f\left(x, t, M_{q}\right)\right]-\mu\left(M_{q-1}-M_{q}\right) \leq 0$ in $\Omega_{\infty}$.

Thus, $M_{q} \geq M_{q+1}$. By condition (D) and (5.2),

$$
\left(L_{k}-\mu\right)\left(M_{q+1}-\gamma\right)=-f\left(x, t, M_{q}\right)-\mu\left(M_{q}-\gamma\right)-L_{k} \gamma \leq 0 \text { in } \Omega_{\infty} .
$$

Since

$\left(M_{q+1}-\gamma\right)(0, t)=-\gamma(0, t) \geq 0, \quad\left(M_{q+1}-\gamma\right)(a, t)=-\gamma(a, t) \geq 0, \quad-\infty<t<\infty$,

we have $M_{j+1} \geq \gamma$. By the principle of mathematical induction, (5.3) follows. 
The sequence $\left\{M_{j}: j=0,1,2, \ldots\right\}$ is monotone nonincreasing and uniformly bounded. Thus, there exists a $T$-periodic function $M(x, t)$ such that $\lim _{j \rightarrow \infty} M_{j}(x, t)$ $=M(x, t)$. Since $M_{j}$ is a $T$-periodic solution of the linear problem, it follows from conditions (i)-(ii) and an argument similar to that in the proof of Theorem 9 that

$$
-f\left(x, t, M_{j}\right)+\mu\left(M_{j+1}-M_{j}\right)
$$

as a function of $x$ and $t$ satisfies the hypotheses for $\Psi$ in Theorem 5. Hence,

$$
M_{j+1}(x, t)=\int_{0}^{T} \int_{0}^{a} G(x, t ; \xi, \tau)\left[f\left(\xi, \tau, M_{j}\right)-\mu\left(M_{j+1}-M_{j}\right)\right] d \xi d \tau .
$$

Since $M_{j+1}$ is $T$-periodic, it suffices to consider $t$ in [0,T]. From (5.4),

$$
\begin{aligned}
M_{j+1}(x, t)= & \int_{0}^{t} \int_{0}^{a} \sum_{n=1}^{\infty} E_{n 1}(t-\tau) \xi^{b} \phi_{n}(\xi) \phi_{n}(x)\left[f\left(\xi, \tau, M_{j}\right)-\mu\left(M_{j+1}-M_{j}\right)\right] d \xi d \tau \\
& +\int_{t}^{T} \int_{0}^{a} \sum_{n=1}^{\infty} E_{n 1}(T+t-\tau) \xi^{b} \phi_{n}(\xi) \phi_{n}(x) \\
& \times\left[f\left(\xi, \tau, M_{j}\right)-\mu\left(M_{j+1}-M_{j}\right)\right] d \xi d \tau . \quad \text { (5.5) }
\end{aligned}
$$

For any fixed $(x, t)$, let $P_{1, j}(\xi, \tau)$ and $P_{2, j}(\xi, \tau)$ denote the first integrand and the second integrand in (5.5), respectively. By Lemma 1 (i) and (ii),

$$
\begin{gathered}
\left|P_{1, j}(\xi, \tau)\right| \leq k_{1} k_{2} \sum_{n=1}^{\infty} \lambda_{n}^{1 / 4} E_{n 1}(t-\tau) \xi^{b / 2}\left[f\left(\xi, \tau, M_{j}\right)-\mu\left(M_{j+1}-M_{j}\right)\right], \\
\left|P_{2, j}(\xi, \tau)\right| \leq k_{1} k_{2} \sum_{n=1}^{\infty} \lambda_{n}^{1 / 4} E_{n 1}(T+t-\tau) \xi^{b / 2}\left[f\left(\xi, \tau, M_{j}\right)-\mu\left(M_{j+1}-M_{j}\right)\right] .
\end{gathered}
$$

Since $M_{j}$ is bounded for any nonnegative integer $j$, we have from conditions (i) and (ii) that $\xi^{b / 2} f\left(\xi, \tau, M_{j}\right)$ is bounded. Because $\phi_{n}(\xi)$ contains a factor $\xi^{\nu}$, and $\frac{b}{2}=\frac{1}{2}-\nu$, it follows that $\xi^{b / 2} M_{0}(\xi, \tau)$ is bounded. From condition (D), and

$$
\xi^{b / 2} \gamma \leq \xi^{b / 2} M_{j} \leq \xi^{b / 2} M_{0}
$$

we have $\xi^{b / 2}\left(M_{j+1}-M_{j}\right)$ being bounded. Therefore for any nonnegative integer $j$, there exists a constant $k_{24}$ such that

$$
\begin{gathered}
\left|P_{1, j}(\xi, \tau)\right| \leq k_{1} k_{2} k_{24} \sum_{n=1}^{\infty} \lambda_{n}^{1 / 4} E_{n 1}(t-\tau), \\
\left|P_{2, j}(\xi, \tau)\right| \leq k_{1} k_{2} k_{24} \sum_{n=1}^{\infty} \lambda_{n}^{1 / 4} E_{n 1}(T+t-\tau) .
\end{gathered}
$$

By (2.12), (3.8), and the Monotone Convergence Theorem,

$$
\int_{0}^{t} \int_{0}^{a} \sum_{n=1}^{\infty} \lambda_{n}^{1 / 4} E_{n 1}(t-\tau) d \xi d \tau \leq a \sum_{n=1}^{\infty} \lambda_{n}^{1 / 4} /\left\{\left(\lambda_{n}-k\right)\left(1-\exp \left[-\left(\lambda_{n}-k\right) T\right]\right)\right\}<\infty .
$$

Similarly by (2.12), (3.9), and the Monotone Convergence Theorem,

$$
\int_{t}^{T} \int_{0}^{a} \sum_{n=1}^{\infty} \lambda_{n}^{1 / 4} E_{n 1}(T+t-\tau) d \xi d \tau<\infty
$$


Hence for any nonnegative integer $j, P_{1, j}(\xi, \tau)$ and $P_{2, j}(\xi, \tau)$ are bounded by integrable functions, respectively. Since $M_{j}(x, t) \rightarrow M(x, t)$ as $j \rightarrow \infty$, it follows that as $j \rightarrow \infty$, $P_{1, j}(\xi, \tau)$ and $P_{2, j}(\xi, \tau)$ converge a.e. to

$$
\begin{gathered}
\sum_{n=1}^{\infty} E_{n 1}(t-\tau) \xi^{b} \phi_{n}(\xi) \phi_{n}(x) f(\xi, \tau, M), \\
\sum_{n=1}^{\infty} E_{n 1}(T+t-\tau) \xi^{b} \phi_{n}(\xi) \phi_{n}(x) f(\xi, \tau, M),
\end{gathered}
$$

respectively on $\Omega^{-}$. It follows from (5.5) and the Lebesgue Convergence Theorem that for any fixed $(x, t)$ in $\Omega^{-}$,

$$
\begin{aligned}
M(x, t)= & \int_{0}^{t} \int_{0}^{a} \sum_{n=1}^{\infty} E_{n 1}(t-\tau) \xi^{b} \phi_{n}(\xi) \phi_{n}(x) f(\xi, \tau, M) d \xi d \tau \\
& +\int_{t}^{T} \int_{0}^{a} \sum_{n=1}^{\infty} E_{n 1}(T+t-\tau) \xi^{b} \phi_{n}(\xi) \phi_{n}(x) f(\xi, \tau, M) d \xi d \tau .
\end{aligned}
$$

Hence, $M(x, t)$ is a $T$-periodic solution of the integral equation (4.1).

Since $M(x, t)$ is bounded, it follows from an argument as in the proof of Theorem 5 that we can interchange summation and integration, and that the series converges uniformly. Thus, $M(x, t)$ is continuous on $\Omega^{-}$. By Dini's Theorem, the sequence $\left\{M_{j}: j=0,1,2, \ldots\right\}$ converges uniformly with respect to $x$ and $t$ to $M(x, t)$.

For any $T$-periodic solution $u(x, t) \geq \gamma(x, t)$, we have from condition $(\mathrm{F})$,

$$
L_{k}\left(M_{0}-u\right) \leq 0 \text { in } \Omega_{\infty} .
$$

By Lemma $4, M_{0} \geq u$. From (5.1) and $M_{0} \geq u$, we have

$$
\left(L_{k}-\mu\right)\left(M_{1}-u\right)=-\left[f\left(x, t, M_{0}\right)-f(x, t, u)\right]-\mu\left(M_{0}-u\right) \leq 0 \text { in } \Omega_{\infty} .
$$

Hence, $M_{1} \geq u$. Let us assume that $u \leq M_{j} \leq \cdots \leq M_{0}$ for some nonnegative integer $j$. By $(5.1)$,

$$
\left(L_{k}-\mu\right)\left(M_{j+1}-u\right) \leq 0 \text { in } \Omega_{\infty},
$$

and hence, $M_{j+1} \geq u$. It follows from the principle of mathematical induction that

$$
\gamma \leq u \leq M_{j+1} \leq M_{j} \leq \cdots \leq M_{0}
$$

for any nonnegative integer $j$, and hence $u \leq \lim _{j \rightarrow \infty} M_{j}=M$.

A proof similar to that of Theorem 11 gives the following result.

THEOREM 12. Suppose conditions (D)-(G) hold. Then, the sequence $\left\{m_{j}(x, t)\right\}$ constructed by $m_{0}(x, t)=\gamma(x, t)$, and for nonnegative integer $j$,

$$
\begin{gathered}
L_{k} m_{j+1}=-f\left(x, t, m_{j}\right)+\mu\left(m_{j+1}-m_{j}\right) \quad \text { in } \Omega_{\infty}, \\
m_{j+1}(0, t)=0=m_{j+1}(a, t), \quad-\infty<t<\infty,
\end{gathered}
$$

satisfies the inequalities:

$$
k_{22} \leq \gamma=m_{0} \leq m_{j} \leq m_{j+1} \leq \cdots \leq M_{0} \leq k_{23} \text { on } \Omega_{\infty}^{-} ;
$$


furthermore, $\left\{m_{j}\right\}$ converges uniformly to $m ; m$ is continuous on $\Omega_{\infty}^{-}$, and is a $T$ periodic solution of the integral equation (4.1). Also $m \leq u$, where $u$ is a $T$-periodic solution of the problem (1.5) and (1.2) with $u \geq \gamma$.

From condition (ii) (a), we have

$$
\left|x^{b / 2}\left[f\left(x, t_{1}, z_{1}\right)-f\left(x, t_{2}, z_{2}\right)\right]\right| \leq Q^{t}\left(k_{22}, k_{23}\right)\left|t_{1}-t_{2}\right|+Q^{z}\left(k_{22}, k_{23}\right)\left|z_{1}-z_{2}\right|
$$

for $\left(x, t_{1}, z_{1}\right)$ and $\left(x, t_{2}, z_{2}\right) \in \Omega_{\infty}^{-} \times\left[k_{22}, k_{23}\right]$. It follows that there exists a Lipschitz constant $Q_{1}$ such that $Q_{1} \leq Q^{z}\left(k_{22}, k_{23}\right)$, and

$$
\left|x^{b / 2}[f(x, t, u)-f(x, t, v)]\right| \leq Q_{1}|u-v|,
$$

where $u(x, t)$ and $v(x, t)$ lie between $\gamma(x, t)$ and $M_{0}(x, t)$. In order to establish existence for the problem (1.5) and (1.2), we need to impose an additional condition on the Lipschitz constant $Q_{1}$. The following result is a consequence of Lemma 10, and Theorems 9, 11, and 12 .

THEOREM 13. Suppose conditions (D)-(G) hold and $Q_{1}$ in (5.6) satisfies

$$
Q_{1} a^{1 / 2} \beta_{1}<1 \text {. }
$$

Then, $M(x, t)$ and $m(x, t)$ are $T$-periodic solutions of the problem (1.5) and (1.2) such that $M \geq u$ and $m \leq u$ for any $T$-periodic solution $u \geq \gamma$.

The proof of the following uniqueness result is similar to that of Theorem 3 of Chan [3].

THEOREM 14. Under the hypotheses of Theorem 13, if condition $(\mathrm{H})$ holds, then each of the sequences $\left\{M_{j}\right\}$ and $\left\{m_{j}\right\}$ converges uniformly to the unique $T$-periodic solution $u(x, t)(\geq \gamma(x, t))$ of the problem (1.5) and (1.2).

Proof. It suffices to show uniqueness. We have $M \geq m$. By condition $(\mathrm{H})$,

$$
L_{k}(m-M) \leq 0 \text { in } \Omega_{\infty} .
$$

Since

$$
(m-M)(0, t)=0=(m-M)(a, t), \quad-\infty<t<\infty,
$$

it follows from Lemma 4 that $m \geq M$. Therefore $M=m$.

Let us consider the more general problem (1.6) subject to (1.4). We impose conditions $(A)$ and $(D)-(H)$. If the boundary conditions are nonhomogeneous, then in addition, we impose $b>-1$, and condition $(C)$ together with the following condition:

(I) $g_{1}(t) \geq \gamma(0, t)$ and $g_{2}(t) \geq \gamma(a, t)$.

Let us construct $\bar{M}_{0}(x, t)$ by

$$
\begin{aligned}
& L_{c} \bar{M}_{0}=-\sigma(x, t) \quad \text { in } \Omega_{\infty}, \\
& \bar{M}_{0}(0, t)=g_{1}(t), \quad \bar{M}_{0}(a, t)=g_{2}(t), \quad-\infty<t<\infty,
\end{aligned}
$$

it follows from Corollaries 7 and 8 that $\bar{M}_{0}(x, t)$ exists and is unique. Also from conditions (D), (F) and (I), and Lemma 4 , we have $\bar{M}_{0} \geq \gamma$. Let

$$
\begin{gathered}
k_{25}=\min \left\{\gamma(x, t):(x, t) \in \Omega^{-}\right\}, \quad k_{26}=\max \left\{\bar{M}_{0}(x, t):(x, t) \in \Omega^{-}\right\}, \\
\beta_{2}=\max \left\{\left[H_{T}(t)\right]^{-1}: t \in[0, T]\right\}, \quad \beta_{3}=\max \left\{\left[H_{T}(t)\right]: t \in[0, T]\right\} .
\end{gathered}
$$


By conditions $(\mathrm{G})$ and $(\mathrm{E})$, there exists a constant $\mu_{1}$ such that (a) if $k_{25} \leq v(x, t) \leq$ $u(x, t) \leq k_{26}$, then

$$
-[f(x, t, u(x, t))-f(x, t, v(x, t))] \leq \mu_{1}[u(x, t)-v(x, t)],
$$

and (b) if $k_{25} \leq u(x, t) \leq k_{26}$ and $u(x, t) \geq \gamma(x, t)$, then

$$
-f(x, t, u(x, t)) \leq \mu_{1}[u(x, t)-\gamma(x, t)] .
$$

Let us construct the sequences $\left\{\bar{M}_{j+1}(x, t)\right\}$ and $\left\{\underline{m}_{j+1}(x, t)\right\}$ with $\underline{m}_{0}(x, t)=\gamma(x, t)$ as follows: for nonnegative integer $j$,

$$
\begin{aligned}
& L_{c} \bar{M}_{j+1}=-f\left(x, t, \bar{M}_{j}\right)+\mu_{1}\left(\bar{M}_{j+1}-\bar{M}_{j}\right) \text { in } \Omega_{\infty}, \\
& \bar{M}_{j+1}(0, t)=g_{1}(t), \quad \bar{M}_{j+1}(a, t)=g_{2}(t), \quad-\infty<t<\infty \text {; } \\
& L_{c} \underline{m}_{j+1}=-f\left(x, t, \underline{m}_{j}\right)+\mu_{1}\left(\underline{m}_{j+1}-\underline{m}_{j}\right) \text { in } \Omega_{\infty}, \\
& \underline{m}_{j+1}(0, t)=g_{1}(t), \quad \underline{m}_{j+1}(a, t)=g_{2}(t), \quad-\infty<t<\infty .
\end{aligned}
$$

From condition (ii) (a), there exists a Lipschitz constant $Q_{2}$ such that $Q_{2} \leq$ $Q^{z}\left(k_{25}, k_{26}\right)$, and for $u(x, t)$ and $v(x, t)$ lying between $\gamma(x, t)$ and $\bar{M}_{0}(x, t)$,

$$
\left|x^{b / 2}[f(x, t, u)-f(x, t, v)]\right| \leq Q_{2}|u(x, t)-v(x, t)| \text {. }
$$

Let $\beta_{4}$ be $\beta_{1}$ with $k$ being replaced by $\left(\int_{0}^{T} c(s) d s\right) / T$. By Corollaries 7 and 8 , and an argument similar to those in Theorems 11-14, we obtain the following result.

Corollary 15. Suppose conditions (D)-(H) and (A) hold, and $Q_{2}$ in (5.7) satisfies $Q_{2} a^{1 / 2} \beta_{4} \beta_{2} \beta_{3}<1$. Then, every $T$-periodic solution $u$ of the problem (1.6) and (1.4) with $g_{1}(t) \equiv 0 \equiv g_{2}(t)$ such that $u \geq \gamma$ satisfies the inequalities:

$$
\gamma=\underline{m}_{0} \leq \underline{m}_{1} \leq \cdots \leq \underline{m}_{j} \leq \cdots \leq u \leq \cdots \leq \bar{M}_{j} \leq \cdots \leq \bar{M}_{1} \leq \bar{M}_{0} \text { on } \Omega_{\infty}^{-} ;
$$

furthermore, both $\left\{\bar{M}_{j}(x, t)\right\}$ and $\left\{\underline{m}_{j}(x, t)\right\}$ converge uniformly to the unique $T$ periodic solution $u(\geq \gamma)$. If the boundary conditions are nonhomogeneous, then the same conclusions hold, provided that in addition, conditions $(\mathrm{C})$ and $(\mathrm{I})$, and $b>-1$ are assumed.

\section{REFERENCES}

[1] M. Abramowitz and I. A. Stegun, eds., Handbook of Mathematical Functions with Formulas, Graphs and Mathematical Tables, National Bureau of Standards, Applied Mathematics Series 55, Washington, 1972 , pp. 362,364

[2] S. B. Chae, Lebesgue Integration, Marcel Dekker, New York, 1980, pp. 216-217, 227-228

[3] C. Y. Chan, Temperatures of linearly and nonlinearly radiating semi-infinite rods, Quart. Appl. Math. 34, 173-182 (1976)

[4] C. Y. Chan and C. S. Chen, A numerical method for semilinear singular parabolic quenching problems, Quart. Appl. Math. 47, 45-57 (1989)

[5] C. Y. Chan and B. M. Wong, Periodic solutions of parabolic problems, to appear in Differential Equations and Applications

[6] B. P. Liu and C. V. Pao, Periodic solutions of coupled semilinear parabolic boundary value problems, Nonlinear Anal. 6, 237-252 (1982)

[7] N. W. McLachlan, Bessel Functions for Engineers, 2nd ed., Oxford at the Clarendon Press, London, 1955, pp. 26, 102-104, 116, 192

[8] M. H. Protter and H. F. Weinberger, Maximum Principles in Differential Equations, Prentice-Hall, New Jersey, 1967, pp. 170, 172 
[9] H. L. Royden, Real Analysis, 2nd ed., Macmillan, New York, 1968, pp. 84, 88, 269

[10] G. N. Watson, $A$ Treatise on the Theory of Bessel Functions, 2nd ed., Macmillan, New York, 1944, pp. 490-492, 506, 593

[11] H. F. Weinberger, A First Course in Partial Differential Equations, Xerox College Publishing, Lexington, 1965 , p. 73

[12] A. H. Zemanian, Distribution Theory and Transform Analysis, McGraw-Hill, New York, 1965, pp. $316-322,333$ 